Submitted to ApJ October 15, 2018

\title{
A Metal-Strong and Dust-Rich Damped Ly $\alpha$ Absorption System toward the Quasar SDSS J115705.52+615521.7
}

\author{
Jian-Guo Wang ${ }^{1,2,3,4}$, Hong-Yan Zhou ${ }^{2,5}$, Jian Ge $^{6}$, Peng Jiang ${ }^{2, *}$, Hong-Lin Lu ${ }^{2}$, J. Xavier \\ Prochaska $^{7}$, Fred Hamann ${ }^{6}$, Hui-Yuan Wang ${ }^{2}$,Ting-Gui Wang ${ }^{2}$ and WeiMin Yuan ${ }^{8}$
}

\begin{abstract}
We report the discovery of an unusual, extremely dust-rich and metal-strong damped Ly $\alpha$ absorption system (DLA) at a redshift $z_{a}=2.4596$ toward the quasar SDSS J115705.52+615521.7 (hereafter J1157+6155) with an emissionline redshift $z_{e}=2.5125$. The quasar spectrum, taken in the Sloan Digital Sky Survey (SDSS), shows a very red color and a number of metal absorption lines, including CII, Al II, Si II, FeII and ZnII, which are confirmed and further characterized by follow-up spectroscopy made with the Multiple Mirror Telescope (MMT). Its neutral hydrogen column density $N_{H I}=10^{21.8 \pm 0.2} \mathrm{~cm}^{-2}$ is among the highest values measured in quasar DLAs. The measured metal column density is $N_{Z n I I} \approx 10^{13.8} \mathrm{~cm}^{-2}$, which is about 1.5 times larger than the largest value in any previously observed quasar DLAs. We derive the extinction curve of the
\end{abstract}

\footnotetext{
${ }^{1}$ National Astronomical Observatories/Yunnan Observatory, Chinese Academy of Sciences, P.O. Box 110, Kunming, Yunnan 650011, China; wangjg@ynao.ac.cn

${ }^{2}$ Key Laboratory for Research in Galaxies and Cosmology, Department for Astronomy, the University of Sciences and Technology of China, Chinese Academy of Sciences, Hefei, Anhui 230026, China;

${ }^{3}$ Key Laboratory for the Structure and Evolution of Celestial Objects, Chinese Academy of Sciences

${ }^{4}$ Graduate School of the Chinese Academy of Sciences, 19A Yuquan Road, P.O. Box 3908, Beijing 100039, China

${ }^{5}$ Polar Research Institute of China, Jinqiao Rd. 451, Shanghai, 200136, China

${ }^{6}$ Astronomy Department, University of Florida, 211 Bryant Space Science Center, P. O. Box 112055, Gainesville, FL 32611, USA

${ }^{7}$ Department of Astronomy and Astrophysics, UCO/Lick Observatory, University of California, 1156 High Street, Santa Cruz, CA 95064, USA

${ }^{8}$ National Astronomical Observatories, Chinese Academy of Sciences, Beijing 100012, China

${ }^{*}$ LAMOST Fellow
} 
dusty DLA using a new technique, which is an analog of the "pair method" widely used to measure extinction curves in the Milky Way (MW). The best-fit curve is a MW-like law with a significant broad feature centered around $2175 \AA$ in the rest frame of the absorber. The measured extinction $A_{V} \approx 0.92 \mathrm{mag}$ is unprecedentedly high in quasar DLAs. After applying an extinction correction, the $i$ band absolute magnitude of the quasar is as high as $M_{i} \approx-29.4$ mag, placing it one of the most luminous quasars ever known. The large gas-phase relative abundance of $[\mathrm{Zn} / \mathrm{Fe}] \approx 1.0$ indicates that metals are heavily depleted onto dust grains in the absorber. The dust depletion level is between that of the warm and cool clouds in the MW. This discovery is suggestive of the existence of a rare yet important population of dust-rich DLAs with both high metallicities and high column densities, which may have significant impact on the measurement of the cosmic evolution of neutral gas mass density and metallicity.

Subject headings: quasars: absorption lines - galaxies: abundances - galaxies: ISM: dust, extinction — quasars: individual: SDSS J115705.52+615521.7

\section{Introduction}

High luminosity quasars are a very useful probe into the early Universe. Gas intervening between us and a distant quasar will leave imprints of absorption lines in the quasar spectrum.

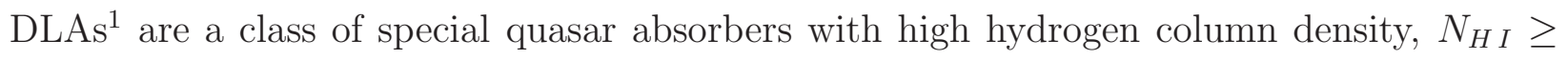
$10^{20.3} \mathrm{~cm}^{-2}$, by definition (see Wolfe et al. 2005 for a comprehensive review). Because of the high column density, gas in DLAs should be mainly neutral under the illumination of background radiation from quasars and galaxies at high redshift (e.g. Prochaska \& Wolfe 1996). DLAs are usually considered as the main neutral gas reservoir for star formation in the high redshift Universe (e.g. Nagamine, Springel \& Hernquist 2004; Prochaska, Herbert-Fort \& Wolfe 2005).

For a quasar at a redshift $z$, its absorption distance (Bahcall \& Peebles 1969) is defined as

$$
X(z)=\int_{0}^{z} \frac{\left(1+z^{\prime}\right)^{2}}{\sqrt{\left(1+z^{\prime}\right)^{2}\left(1+z^{\prime} \Omega_{m}\right)-z^{\prime}\left(z^{\prime}+2\right) \Omega_{\Lambda}}} d z^{\prime}
$$

In principle, we may calculate the mean co-moving $\mathrm{H}$ I density by averaging $N_{H}$ in different

\footnotetext{
${ }^{1}$ In this paper, this abbreviation is referred to as quasar DLAs unless further specified.
} 
sight lines,

$$
\Omega_{H I}(X) d X=\frac{\mu m_{H} H_{0}}{c \rho_{c}} \int_{N_{m i n}}^{\infty} N_{H I} f\left(N_{H I}, X\right) d N_{H I},
$$

where $\mu=1.3$ is the mean molecular weight of absorption gas, $m_{H}$ the hydrogen atomic mass, $H_{0}$ the Hubble constant, $\rho_{c}$ the critical mass density, and $f\left(N_{H I}, X\right)$ the neutral hydrogen column density distribution of quasar absorbers. The observed $f\left(N_{H I}, X\right)$ can be crudely described either by a $\Gamma$ function (Fall \& Pei 1993; Péroux et al. 2003) or by a double power-law with a break at $\log \left(N_{H I} / \mathrm{cm}^{-2}\right) \sim 21.5$ (Prochaska \& Wolfe 2009; Noterdaeme et al. 2009).

Although great efforts have been made to measure the neutral hydrogen column density, $N_{H I}$, and frequency distribution, $f\left(N_{H I}, X\right)$, of DLAs, the slope at the high $N_{H I}$ end is not well constrained by observations. The slope varies from $\alpha \sim-6.0$ (Prochaska \& Wolfe 2009) to $\alpha \sim-3.5$ (Noterdaeme et al. 2009), unlike the stable measurement of slope at the low $N_{H I}$ end, $\alpha \sim-2$ (Péroux et al. 2005; Prochaska \& Wolfe 2009; Noterdaeme et al. 2009). Such a disagreement is likely originated from the large statistical fluctuation caused by the rare detections of high $N_{H I}$ DLAs. Since the mean co-moving H I density, $\Omega_{H}(X)$ (Bahcall \& Peebles 1969), depends sensitively on the slope of $f\left(N_{H I}, X\right)$ at high $N_{H} 2$, the uncertainty (of the slope) leaves the measurement of $\Omega_{H I}(X)$ inaccurate. It is quite desirable to measure more high column density DLAs to determine the shape of $f\left(N_{H I}, X\right)$ at the high $N_{H I}$ end correctly. However, so far, only two super-DLAs with $\log \left(N_{H I} / \mathrm{cm}^{-2}\right) \sim 22$ have been identified, SDSS J081634.40+144612.9 (hereafter J0816+1446; Noterdaeme et al. 2009; Guimarães et al. 2012) and SDSS J113520.39-001052.5 (hereafter J1135-0010; Kulkarni et al. 2012; Noterdaeme et al. 2012).

The paucity of high $N_{H}$ I DLA detections can be attributed either to their intrinsic rarity or to selection effect induced by dust obscuration or both. Early evidence for dust in DLAs was found by comparison of the continuum slope between quasars with and without DLAs (e.g. Fall \& Pei 1989; Fall, Pei \& McMahon 1989; Pei, Fall \& Bechtold 1991), which was later confirmed by the studies of the differential depletions between refractory and volatile elements (e.g. Pettini et al. 1994, 1997; Vladilo et al. 2002). Currently, only two dust-rich $\operatorname{DLAs}\left(A_{V} \approx 0.2\right.$ in SDSS J0918+1636, Fynbo et al. 2011 ; and $A_{V} \approx 0.4$ in AO $0235+164$, Junkkarinen et al. 2004) have been reported. On the contrary, dusty absorbers are detected frequently in other astrophysical environments. In the MW, dusty clouds with $A_{V} \sim 1.0 \mathrm{mag}$ are commonly seen (Diplas \& Savage 1994). High dust extinction was measured in the local galaxies as well (e.g. Xiao et al. 2012). Even at high redshift, many very dusty absorbers have been detected by observations of GRB afterglow (e.g. Schady et al. 2011). Fall \& Pei

\footnotetext{
${ }^{2} f\left(N_{H I}, X\right)$ is extrapolated to infinity when taking the integral in Equation (2).
} 
(1993) developed an analytical model to study the selection effect due to the obscuration of dust in DLAs and suggested that a fairly large fraction of dusty DLAs has been overlooked in the quasar surveys. The full obscuration model requires a luminosity function of quasars, a distribution of dust-to-gas ratio and a distribution of $N_{H I}$ in DLAs. The application of the obscuration model is presented in $\S 5$.

Star formation leads to consumption of neutral gas and enrichment of heavy elements. The measurements of average metallicity at different redshifts provide robust diagnosis for cosmic star formation. The DLA technique could be a powerful tool to probe the metals in the high redshift Universe. However, current measurements suggest that DLAs are generally metal-poor. The mean metallicity is only a few percents of the solar abundance (e.g., Kulkarni \& Fall 2002; Prochaska et al. 2003; Kaplan et al. 2010). It is significantly lower than the metallicity measured in the MW and the nearby galaxies as well as the high redshift galaxies selected through their starlight emissions, such as Lyman break galaxies (e.g., Giavalisco 2002 and references therein). Since dust grains are made of heavy elements, the correlation between metallicity and dust abundance is expected (e.g., Xiao et al. 2012). Therefore, a direct explanation for the deficiency of heavy elements in DLAs is that the metal-rich absorbers suffer relatively large dust obscuration and thus are systematically omitted in the DLA surveys (e.g., Boissé et al. 1998).

In this paper, we report the discovery of an unusual DLA with high column density $N_{H I}=10^{21.8 \pm 0.2} \mathrm{~cm}^{-2}$ toward the quasar J1157+6155. It is the metal-strongest and dustrichest quasar DLA to the best of our knowledge. We further characterized the property of its dust content by evaluating the extinction curve in the absorption rest frame. The resulted curve exhibits a significant $2175 \AA$ extinction bump, which is very rarely seen in quasar absorption line systems (e.g., Wang et al. 2005; Noterdaeme et al. 2008; Zhou et al. 2010; Jiang et al. 2011). The paper is organized as follows. In $\S 2$, we describe the data used in this paper, including archived data and our MMT spectroscopic data. We evaluate the extinction curve in $\S 3$ and measure the absorption line spectrum in $\S 4$. The results are summarized and discussed in $\S 5$. Throughout this paper, we adopt the cometic abundances from Asplund et al. (2009) as solar abundances, and assume a cosmology with $H_{0}=70 \mathrm{~km} \mathrm{~s}^{-1} \mathrm{Mpc}^{-1}, \Omega_{\mathrm{M}}=0.3$, and $\Omega_{\Lambda}=0.7$.

\section{Observations and Data Reduction}

J1157+6155 was imaged in the SDSS on May 18, 2001 in the $u, g, r, i$, and $z$ bands, giving point-spread-function (PSF) magnitudes of 24.58 $\pm 1.23,20.69 \pm 0.03,19.69 \pm 0.03,19.24 \pm 0.02$, and $18.47 \pm 0.04$ (AB-system), respectively. J1157+6155 was selected as a high-redshift 
quasar candidate based on its location in the 'griz color' cube (Richards et al. 2004), and was confirmed as a quasar at an emission line redshift $z_{e}=2.5125 \pm 0.0013$ by an spectroscopic observation performed on Feb. 15, 2002. The SDSS spectrum, extracted from the SDSS data release 7 (DR7; Abazajian et al. 2009), is very red with a number of absorption lines imposed. We identified a broad absorption feature in the blue end of the spectrum as a DLA at the same redshift as those of the metal absorption lines $\left(z_{a}=2.4596\right)$. Of particular interest are very strong absorption features at the $\lambda 2026 \mathrm{Zn} \mathrm{II}+\mathrm{Mg}$ I blend and the $\lambda 2062$ Zn II + Cr II blend with rest-frame equivalent widths $(\mathrm{EW})$ of $\sim 1 \AA$, which have never been seen in any previous quasar DLAs, as far as we know.

To investigate J1157+6155 in more detail, we performed follow-up spectroscopic observations using the Blue Channel Spectrograph mounted on the 6.5 m MMT. Two exposures with length of $1200 \mathrm{~s}$ were taken by using the $500 \mathrm{~g} \mathrm{~mm}^{-1}$ grating on February 11, 2008. Another exposure with length of $900 \mathrm{~s}$ was obtained by using the $800 \mathrm{~g} \mathrm{~mm}^{-1}$ grating on March 30, 2008. A slit width of $1^{\prime \prime} .0$ was chosen to match the seeing in both of the nights. The $500 \mathrm{~g} \mathrm{~mm}^{-1}$ grating was blazed at $6000 \AA$ (1734 $\AA$ in the DLA rest frame) and the $800 \mathrm{~g} \mathrm{~mm}^{-1}$ grating at $4000 \AA$ (1156 $\AA$ in the DLA rest frame). They provide a wavelength coverage of $\lambda \sim 4400-7500 \AA(1272-2168 \AA$ in the DLA rest frame) and $\lambda \sim 3200-5200 \AA$ (925 - $1503 \AA$ in the DLA rest frame), respectively. The corresponding spectral resolutions are 3.8 and $2.5 \AA$ in full width at half maximum (FWHM) measured from the arc lamp lines. The CCD reductions, including bias subtraction, flat-field correction, and cosmic ray removal, were accomplished with the standard procedures using IRAF3. Wavelength calibration was carried out using $\mathrm{He} / \mathrm{Ne} / \mathrm{Ar}$ lamp. A KPNO standard star was observed for flux calibration.

J1157+6155 was also detected on March 1, 1993 during the Two Micron All Sky Survey (2MASS; Skrutskie et al. 2006) with magnitudes (Vega-system) of $16.894 \pm 0.183,16.314 \pm$ 0.202 and $15.093 \pm 0.155$ in the $J, H$ and $K_{s}$ band, respectively. All of the photometric and spectrophotometric data were first corrected for Galactic reddening using the extinction map of Schlegel et al. (1998) and the reddening curve of Fitzpatrick (1999) before performing further analysis. The three MMT spectra, after scaled by a small factor to compensate for the aperture effect and the uncertainty of absolute flux calibration, are consistent with the SDSS spectrum in the overlapped wavelength range. The SDSS and MMT spectra are combined to form one spectrum covering a wavelength range of $3200-9200 \AA(925-2660 \AA$ in the DLA rest frame). The combined spectrum is used to derive an extinction curve in $\S 3$.

\footnotetext{
${ }^{3}$ IRAF is distributed by the National Optical Astronomy Observatory, which is operated by the Association of Universities for Research in Astronomy, Inc., under cooperative agreement with the National Science Foundation.
} 


\section{Reddening and Extinction Curve}

In this section, we explore the distribution of the spectral indices of quasars and derive the extinction curve of the DLA with two methods.

\subsection{Distribution of Quasar Spectral Indices}

The rest-frame UV to optical spectral energy distribution (SED) of the quasar J1157+6155 is extremely red, suggesting that it is heavily reddened by the foreground DLA. To gauge the dust extinction of $\mathrm{J} 1157+6155$, we first examined the distribution of spectral indices $\alpha$ of SDSS quasars in the redshift interval $2.4<z<2.6$, which is defined as $S_{\lambda} \propto \lambda^{\alpha}$ and calculated in two continuum windows (1690-1720 and $2225-2250 \AA)$ in the quasar rest frame. The distribution function is roughly a Gaussian with a red "tail" (see Fig. 1). The red "tail" is similar to the red "tail" of the color distribution of quasars which was attributed to dust reddening (Hopkins et al. 2004). The spectrum of J1157+6155 is very red, with an index $\alpha \approx 0.24$, that deviates significantly from the vast majority of the distribution. This motivates us to model its extinction curve in detail.

\subsection{Overview of the Modeling Methods}

Light from a quasar behind an intervening DLA is subject to wavelength-dependent extinction by dust therein. The extinction curve can be characterized by comparing the observed spectra with the intrinsic one, which are usually unknown. A composite spectra of quasars, scaled by a factor, is often used as a substitute to the intrinsic spectra (e.g. Wang et al. 2004; Zhou et al. 2010). However, the feasibility of using composite quasar spectra is questionable because of the rich diversity of quasar spectra. In order to alleviate this problem, we design a new method to derive extinction curves and to gauge the uncertainties induced by mismatches among the intrinsic quasars spectra. We first build up a spectra library of quasar as templates to model the intrinsic spectrum of a target. The observed target spectrum is then fitted with models as the template spectra drawn from the library that are reddened by dust with a parameterized extinction curve. These fittings with different template spectra result in distributions of the best-fit parameters, which are in turn used to derive the best-estimates of the parameters and their systematic uncertainties due to mismatches among the templates. We refer to this method as "quasar spectra pair method". 


\subsection{Composite Quasar Spectrum Method}

A parameterized method for determining extinction curves was described by Zhou et al. (2010) and Jiang et al. (2010a,b; 2011). The spectrum of a target is modeled by a composite quasar spectrum, which is reddened with a parameterized extinction curve. The best-fit parameters are determined by minimizing $\chi^{2}$ using MPFIT (Markwardt 2009). We use the parameterized UV/optical extinction curve of Fitzpatrick \& Massa (1990, 2007),

$$
k(\lambda)=c_{1}+c_{2} x+c_{3} D\left(x, x_{0}, \gamma\right)
$$

where $x \equiv \lambda^{-1}$, in units of inverse microns $\left(\mu m^{-1}\right)$. There are five free parameters in the formula which correspond to two features in the curve: (1) a linear component underlying the entire UV wavelength range, with two parameters $c_{1}$ and $c_{2} ;(2)$ a Lorentzian-like $2175 \AA$ bump (e.g., Tielens 2005), described by three parameters: strength $c_{3}$, center wavelength $x_{0}$, and width $\gamma$, then expressed as

$$
D\left(x, x_{0}, \gamma\right)=\frac{x^{2}}{\left(x^{2}-x_{0}^{2}\right)^{2}+x^{2} \gamma^{2}}
$$

Following Zhou et al. (2010), the composite quasar spectrum is obtained by combining the SDSS composite spectrum $(\lambda \leq 3000 \AA$; Vanden Berk et al. 2001) and the near-infrared template $(\lambda>3000 \AA$; Glikman et al. 2006). In the fitting procedure, only the wavelength range longer than $1250 \AA$ is used, and small weights are assigned to regions around strong broad emission lines, including Mg II $\lambda 2800$, C III]1909, C IV1549 and Si IV1400, while absorption lines are masked. The best-fit results are $c_{1}=-2.75, c_{2}=0.48, c_{3}=0.43, x_{0}=4.82$, and $\gamma=1.13$. The best-fit model (red line) is overplotted with the observed spectrum in the upper panel of Fig. 2. In the near-infrared band, the resultant model is consistent with 2MASS photometry. We also attempted to fit the observed spectrum using an SMC-like extinction curve (Pei 1992; Gordon et al. 2003). The resultant spectrum disagrees with the observed one around $2000-2200 \AA$ in the DLA rest frame, indicating the requirement of an absorption bump. In the bottom panel of Fig. 2, we compare the SMC-like extinction curve (dotted black line) with the best-fit extinction curve (solid black line). The extinction curve derived here is subject to an arbitrary shift (on $c_{1}$ ) of the composite quasar spectrum (Zhou et al. 2010; Jiang et al. 2011) 4 In fact, a physical extinction curve needs to be zero when it goes toward $\lambda \rightarrow \infty$. Thus, we can physically determine the parameter $c_{1}$ by forcing the extinction to be zero when extrapolating the best-fit extinction curve to $\lambda \rightarrow \infty$. It yields $c_{1}=0$ and $A_{V}=0.88 \mathrm{mag}$.

\footnotetext{
${ }^{4} c_{1}$ depends on the flux level of the composite quasar spectrum, which is arbitrary selected.
} 


\subsection{Quasar Spectra Pair Method}

\subsubsection{Building up the Template Library}

Composite quasar spectra were often used as a substitute to the intrinsic spectra of target quasars in previous work. However, as aforementioned, the feasibility of such substitution is suspect because of the rich diversity of the quasar continuum and pseudo-continuum composed of a large number of Fe II emission lines (Pitman et al. 2000). To explore the systematic uncertainty due to the diversity of quasar spectra, we build up a library of quasar spectra, and use them to model the intrinsic spectrum of target quasars. In this process the parameters of the extinction curves are extracted. The distributions of derived parameters are then used to gauge the systematic uncertainties introduced by the spectral diversity.

The library of quasar spectra in the redshift range of $0.6<z<2.4$, which are selected from the SDSS DR7 quasar catalog (Schneider et al. 2010), is built up in the following steps. Note that we do not build templates with $z_{e} \geq 2.4$ because the number of quasars with high quality SDSS spectra decreases quickly beyond this redshift.

1. Discarding possible Broad absorption line (BAL) quasars by making use of the catalogs of Gibson et al. (2009), Scaringi et al. (2009) and Shen et al. (2011).

2. Calculating the power-law index of quasar continuum. The power-law index is calculated using the continuum windows: $1690-1700 \AA$ and $2225-2250 \AA$ for quasars with redshifts $z \geq 2,2225-2250 \AA$ and $3030-3090 \AA$ for quasars with redshifts $0.8<z<2,3030-3090 \AA$ and $4020-4050 \AA$ for quasars with redshifts $z \leq 0.8$.

3. Selecting objects according to the distribution of the power-law indices. In each redshift interval of $\delta z=0.2$, the distribution of the power-law indices is found to be roughly a Gaussian with a "red tail", as shown in Fig. 1. Quasars in the "red tail" (to the right of the $2 \sigma$ dashed line) may be either intrinsically red or reddened by dust. We assume that the un-reddened intrinsic distribution of the indices is Gaussian. We randomly select quasars from the "red tail" of the distributions while keep the others (on the left of the $2 \sigma$ dashed line), so as to make the resulting distributions of the indices following a Gaussian distribution (similar to the red line in Fig. 1). The first $\sim 500$ quasars with the highest $\mathrm{S} / \mathrm{N}$ ratios are selected in each redshift interval.

4. Removing narrow absorption lines from the selected spectra. Each of the spectra selected (denoted as $f_{0}$ ) is smoothed using the robust local regression smoothing (Cleveland 1979; denoted as $f_{1}$ ), and then the initial spectrum is normalized by its smoothed spectrum, $f_{2}=f_{0} / f_{1}$. For $f_{2}$, pixels with values smaller than 0.85 may be part of 
absorption lines and their values are set to 1. Narrow absorption lines superimposing over broad emission lines, such as Mg II $\lambda 2800$, C III] $\lambda 1909$, C IV $\lambda 1549$ and Si IV $\lambda 1396$, cannot be effectively recovered with this approach. The profile of each of the broad emission lines in the normalized spectrum $f_{2}$ is then fitted by a Gaussian. The fitting result is denoted as $f_{3}$. $f_{2}$ is then re-normalized by $f_{3}, f_{4}=f_{2} / f_{3}$. Similarly, pixels of $f_{4}$ with values smaller than 0.85 are set to 1 . The "true" spectrum of a quasar is the product of $f_{4} \times f_{3} \times f_{1}$.

Finally, all the template spectra are visually inspected to ensure that there are no apparent absorption lines.

\subsubsection{Refined Modeling}

After the library of the intrinsic spectra of quasars is built up, the target spectrum is fitted with the $\sim 500$ template spectra in a redshift interval $\left[z_{e}, z_{e}+0.2\right]$. For J1157+6155, the templates are obtained in the redshift interval $[2.2,2.4]$, since the high redshift template with $z_{e}>2.4$ is not available in the library. Only the common wavelength range of the target spectrum and the template spectra are used in the fit, which is $1250-2620 \AA$ for J1157+6155 in the quasar rest frame. Next, we will extract the intrinsic strength of Fe II emission of quasar J1157+6155 and constrain the extinction curve fitting with only the template quasar spectra having a similar Fe II emission strength. The refined modeling procedure can help to reduce the contamination from FeII emission when measuring the bump profile.

We first take the median of each of the parameters of the extinction curve from fitting the $\sim 500$ templates as the best-fit values, and de-redden the observed spectrum with the median extinction curve. Then we model the Fe II emission of the de-reddened spectra using the method described in Wang et al. (2009) and estimate its EW. The Fe II template used here is a combination of the FeII template of Vestergaard \& Wikes $(2001 ; \lambda<2200 \AA)$ and that of Tsuzuki et al. (2006; $\lambda \geq 2200 \AA)$. Next we measure the Fe II EWs for every quasar in the library in the same way. Only the quasars whose FeII EWs are consistent with that of the target spectrum within $1 \sigma$ uncertainty are selected as the templates for refined modeling. This selection results in $\sim 100$ Fe II matched template spectra. We repeat the extinction curve fitting procedure with the Fe II matched template spectra. Finally, the best-fit parameters and their uncertainties are adopted from the distributions of the fitted parameters, which can be modeled with Gaussians (Fig. 3). They are $c_{2}=0.50 \pm 0.04$, $x_{0}=4.79 \pm 0.04, \gamma=0.98 \pm 0.20$ and $c_{3}=0.37 \pm 0.18$, where the values are the expectations and the errors are the standard deviations. Since $c_{1}$ is an arbitrary parameter depending on the brightness of template spectra as stated above, it is not presented in Fig. 3. 
The significance of the $2175 \AA$ absorption bump can be gauged by the distribution of bump strength (Jiang et al. 2011), which is measured by the area of the bump $A_{\text {bump }}=$ $\pi c_{3} / 2 \gamma$. The red Gaussian (in panel $e$ of Fig. 3) is the distribution of bump strength derived with the Fe II matched template spectra. The expectation is $A_{b u m p}=0.61$ and the standard deviation is $\sigma=0.19$. Thus, the null hypothesis (i.e. no $2175 \AA$ bump or $A_{\text {bump }}=0$ ) can be rejected at a statistical confidence level greater than $3 \sigma$. Alternatively, the $2175 \AA$ absorption bump in $\mathrm{J} 1157+6155$ is detected at a confidence level of $>3 \sigma$. In the same panel, we also present the bump strength distribution derived with the full template library (the blue Gaussian), which is nearly twice broader than that derived with the Fe II matched template spectra. In this case, the $2175 \AA$ absorption bump is only detected at a confidence level of $2 \sigma$. The comparison clearly shows the contamination of the variations of Fe II broad emissions on the measurements of bump strength (Pitman et al. 2000). We conclude that the refined modeling procedure is necessary for detecting relatively weak $2175 \AA$ absorption bumps on quasar spectra. Given the Fe II emissions on the composite quasar spectrum might not match with that on the observed spectrum, the quasar spectra pair method should be more accurate than the composite quasar spectrum method on measuring the $2175 \AA$ absorption bumps in general. The comparison of extinction curve derived with quasar spectra pair method and that of the composite quasar spectrum method is presented in Fig. 4.

The parameter $c_{1}$ is recalculated by forcing the extinction to zero for $\lambda \rightarrow \infty$. This gives $A_{V}=0.92 \pm 0.07 \mathrm{mag}$ and a total-to-selective extinction ratio of $R_{V} \equiv A_{V} / E(B-V) \approx 3.97$. The slope of the underlying extinction is similar to that found in J1007+2853 $\left(R_{V} \approx 3.87\right)$, a very dusty quasar with super-strong $2175 \AA$ absorption (Zhou et al. 2010). Both slopes are larger than the averaged value of $R_{V}=3.1$ in the MW (e.g., Draine 2003). The background quasar is intrinsically very bright with $i \approx 16.5 \mathrm{mag}$ after de-reddening using the derived extinction curve; this corresponds to an intrinsic luminosity of $M_{i} \approx-29.4 \mathrm{mag}$, assuming an optical spectral slope of $\alpha_{\nu}=0.5\left(S_{\nu} \propto \nu^{-\alpha_{\nu}}\right.$; Schneider et al. 2010). We note that the 2MASS $K_{s}$ band absolute magnitude is as high as $M_{K_{s}} \approx-31.5$ even without extinction correction 5 . In addition, the redshift of $2175 \AA$ bump in J1157+6155 z $=2.46$ is slightly higher than the previously highest redshift $2175 \AA$ absorber toward the Gamma Ray Burst (hereafter GRB) 070802 ( $z=2.45$; Elíasdóttir et al. 2009).

\footnotetext{
${ }^{5}$ We assume an optical to near-infrared slope of $\alpha_{\nu}=1\left(S_{\nu} \propto \nu^{-\alpha_{\nu}}\right.$; Glikman et al. 2006).
} 


\section{Column Densities, Gas-Phase Abundances and Dust Depletion}

The Ly $\alpha$ absorption line of J1157+6155 is covered by both SDSS and MMT spectra. We perform line profile fitting on the MMT spectrum, since it has higher signal-to-noise ratio. The fitting is realized by using the program x_fitdla from the XIDL package6. The best-fit Voigt profile, as shown in Fig.5, yields a column density $\log N_{H I}=21.8 \pm 0.2 \mathrm{~cm}^{-2}$. There are additional absorptions blueward of the DLA, which may be attributed to unidentified absorption lines corresponding to absorbers at different redshifts.

In order to measure the column density of metal absorption lines, we normalize the observed spectrum using an absorption-free quasar continuum modeled by a series of polynomials. The normalized spectra are plotted in velocity space in Fig. 6. The Fe $\lambda \lambda 22344$, 2374, 2382, 2586, 2600 absorption lines are taken from SDSS spectrum, because they are not covered by our MMT spectra. The curve of growth (COG) method is employed to measure the absorption line column densities of heavy elements (Jenkins 1986). The equivalent widths (EWs) of all transitions are calculated by integrating over an velocity interval $[-800,400] \mathrm{km} \mathrm{s}^{-1}$, which is so-chosen to cover most of the absorption of the detected transitions and void the contamination from nearby absorptions. The Fe II $\lambda \lambda$ 1608, 2344, 2374, 2382, 2586 and 2600 absorption lines, arising from the ground level of $\mathrm{Fe}^{+}$, are used to derive the COG. The best-fit column density $N_{F e I I}=15.62 \pm 0.19 \mathrm{~cm}^{-2}$ and Doppler parameter $b=182 \pm 26 \mathrm{~km} \mathrm{~s}^{-1}$, are obtained by $\chi^{2}$ minimization. We further explore the $\chi^{2}$ space by stepping the $N_{F e I I}$ and $b$ to construct the contours of the $68 \%$ and $90 \%$ confidence levels. The error-bars reported above are corresponding to the $68 \%$ confidence interval. Using the best-fit COG (Fig. 7), we derive the column densities of other ions. For species having multiple transitions available, the column densities are obtained by fitting all the absorptions simultaneously.

The derived Doppler parameter $b$ from COG is relatively large. It may indicate that the absorption lines have narrow sub-structures, which cannot be resolved on the MMT and SDSS spectra. Thus, the column densities based on the single-component COG might be underestimated (Prochaska 2006). We also measure the column densities of all the absorption lines using the apparent optical depth method (AODM; Savage \& Sembach 1991). The resultant column densities are systematically smaller than that derived by COG method. The difference is expected since the absorption lines are heavily saturated generally. Thus, we adopt the column densities on the basis of COG in the rest of this paper. The measurements are summarized in Table 1.

\footnotetext{
${ }^{6}$ http://www.ucolick.org/ xavier/IDL
} 
The measured $N_{Z n I I}$ of $\mathrm{J} 1157+6155$ is $\sim 1.5$ times higher than that of the previous record holder, SDSS J1137+3907, whose $N_{Z n I I}=(2.692 \pm 0.311) \times 10^{13} \mathrm{~cm}^{-2}$ (Meiring et al. 2006). Since the column density of neutral hydrogen is very large, the ionization correction of metal elements is ignorable. Then, the gas-phase abundances relative to solar values are derived, yielding $[\mathrm{Zn} / \mathrm{H}]=-0.60 \pm 0.38,[\mathrm{Si} / \mathrm{H}]=-0.83 \pm 0.27,[\mathrm{Fe} / \mathrm{H}]=-1.62 \pm 0.28$, $[\mathrm{Cr} / \mathrm{H}]<-1.36,[\mathrm{Ni} / \mathrm{H}]<-1.32 . \mathrm{J} 1157+6155$ is the metal-strongest DLA to the best of our knowledge. In fact, these abundances might be even larger, since the column densities of metal absorption may have been underestimated as stated above. Boissé et al. (1998) noted that all DLAs in their sample lie below an threshold $\left(N_{Z n I I}=1.4 \times 10^{13} \mathrm{~cm}^{-2}\right)$ in the $[\mathrm{M} / \mathrm{H}]-N_{H}$ I plane, and explained that the threshold was set by the dust obscuration bias. A similar trend has been seen in a larger sample of DLAs compiled by Prochaska et al. (2007) as well (see Fig. 8). The lack of metal-strong DLA in the previous surveys may be caused by the disadvantage of conventional quasar selecting methods on the basis of the UV-blue excess. The SDSS uses a quasar selection criterion (Richards et al. 2002) that is more compatible with dust reddened quasars than the conventional methods. Herbert-fort et al. (2006) obtained a sample of metal-strong DLAs having similar metallicity with the MW clouds (Roth \& Blades 1995) in SDSS 8 Kaplan et al. (2010) studied the quasar color of this DLA sample and found significant dust reddening. Among the metal-strong DLAs (filled circles in Fig. 8), J1157+6155 has the highest column density of gas. The population of high column density and metal-strong DLAs, bearing bulks of heavy elements, has great weight on the measurement of the cosmic metallicity.

In Fig. 9, we show comparison between the dust depletion pattern of J1157+6155 and that of the MW cool/warm disk clouds on the line of sight towards $\zeta$ Oph (Savage \& Sembach 1996). The dust depletion pattern of J1157+6155 is quite similar with the MW clouds. The MW-like depletion pattern has been seen in two $2175 \AA$ quasar absorbers previously (Jiang et al. 2010b). The heavy dust depletion confirms the existence of dust grains and indicates that J1157+6155 may be a $H_{2}$-bearing DLA according to the correlation between the presence of $\mathrm{H}_{2}$ and high depletion level (Ge \& Bechtold 1997; Ge et al. 2001; Noterdaeme et al. 2008).

Vladio et al. (2008) derived the average dust-to-gas ratio of DLAs $\left(A_{V} / N_{H I} \approx 3 / 10^{22}\right.$ mag $\mathrm{cm}^{2}$; the dotted curve in Fig. 10), by comparing the color of 248 quasars with DLAs and that of a large non-DLA quasar sample. The dust-to-gas ratio of the three dust-rich DLAs (J0918+1636 and AO 0235+164 as well as J1157+6155 in this work) is larger than the

\footnotetext{
${ }^{7}$ Chemical abundance relative to the solar value defined as $[X / Y] \equiv \log (X / Y)-\log (X / Y) \odot$.

${ }^{8} \mathrm{~J} 1157+6155$ in this work can be classified as a metal-strong DLA according to the definition in Herbertfort et al. (2006).
} 
average value by a factor of 5. While the two super-DLAs (J0816+1446 and J1135-0010) have high column densities of gas but very little dust content. Their dust-to-gas ratio is about $1 / 4$ of the average value of DLAs. We conclude that the dispersion of dust-to-gas ratio in DLAs is quite large and can vary over an order of magnitude.

\section{Discussion}

The uniqueness of J1157+6155 endows it an ideal laboratory to explore DLA properties. Its neutral hydrogen column density of $N_{H I}=10^{21.8 \pm 0.2} \mathrm{~cm}^{-2}$ is the top several among known quasar DLAs. The measured $N_{Z n I I}$ is the highest among quasar DLAs to the best of our knowledge. Combining the SDSS spectroscopic data and the 2MASS photometric data of $\mathrm{J} 1157+6155$, we inferred an extinction as large as $A_{V} \approx 0.92 \mathrm{mag}$, which is much larger than that ever reported in quasar DLAs. The presence of dust grains is further confirmed by the high dust depletion levels measured on our following up MMT spectrum. The discovery of DLA J1157+6155 may reveal the existence of an important population of metal-strong and dust-rich super-DLAs.

This new population of DLAs might be overlooked due to dust obscuration in the previous DLA surveys, which are generally based on optically selected quasar samples. Even if occasionally detected, the metal-strongest DLAs tend to be found at the faint end of quasar brightness distributions in magnitude-limited surveys. Herbert-fort et al. (2006) compiled a sample of metal-strong DLAs and gauged the significance of the correlation between $N_{Z n^{+}}$ and r-magnitude. The correlation is not significant as expected by the authors. We note that the censored data were excluded in their statistical study. By taking the censored data into account and adding two new metal-strong DLAs, J1157+6155 in this work and the one at $z=2.58$ towards the quasar SDSS J0918+1636 reported by Fynbo et al. (2011), we find a marginal correlation between r-magnitude and $N_{Z n^{+}}$with a Spearman coefficient of $r_{S}=0.39$ and a chance probability of $P_{\text {null }}<3.9 \%$ using the ASURV package (Isobe et al. 1986). It suggests that DLAs, at least metal-strong DLAs, are likely to be affected by dust obscuration. Attenuated by very dusty absorbers, even quasars as intrinsically luminous as J1157+6155 - one of the few ten highest luminosity quasars in about one hundred thousand SDSS quasars - could easily escape from quasar DLA surveys. Herbert-fort et al. (2006) adopted a brightness requirement of $r<19.5$ mag when they compiled the metal-strong DLA sample. With $r=19.69 \mathrm{mag}$, J1157+6155 is just escaped from this brightness threshold.

Therefore, the dust-rich DLAs should mainly be detected toward the faint quasars. Deep quasar surveys are desired to disclose the abundance of dust-rich DLAs with both high metallicities and high column densities. Since only intrinsically luminous quasars can be 
observed after intersecting a very dusty absorber, an amplification factor on their number density is necessary to correct the selection effect induced by dust obscuration in magnitude limited quasar surveys. We estimate the ratio of the real number density and the observed number density of dusty DLAs having a similar optical depth and redshift as J1157+6155 on the basis of the dust obscuration model built by Fall \& Pei (1993). By defining $\rho_{t}(\tau, z)$ as the mean numbers of absorbers along random lines of sight with optical depths $\tau$ at a fixed redshift $z$ and $\rho_{o}(\tau, z)$ as the mean numbers along observed lines of sight, the correction factor can be expressed as:

$$
\eta=\rho_{t}(\tau, z) / \rho_{o}(\tau, z)=e^{\tau \beta}
$$

where $\tau$ is the optical depth in $V$ band at $z=0$, and $\beta$ is the negative exponent at the bright end of the quasar luminosity function. For simplicity, we assume that the extinction law is $\tau_{\text {rest }} \propto 1 / \lambda$ in the DLA rest frame. Then the optical depths $\tau$ in the equation (5) yields $\tau=\tau_{\text {rest }}(1+z) \sim A_{V}^{\text {rest }}(1+z)$. Applying the measured $A_{V}^{r e s t}$ and $z$ in the case of J1157+6155 and $\beta \sim 2.1$ from the SDSS quasar luminosity function (Richards et al. 2002), we obtained a huge correction factor, $\eta \sim 1,000$. One serendipitous detection of the dust-rich DLAs in the $\sim 12,000$ high redshift $(z>2.5)$ quasars of SDSS DR7 (Schneider et al. 2010) and the huge amplification factor of observed number density jointly indicate a significant population of dust obscured DLAs 9

The cosmological mean metallicity $\langle Z\rangle$ (i.e., the log of the ratio of the co-moving densities of metals and gas relative to the solar abundance) measured in DLAs can be calculated as:

$$
\langle Z\rangle=\log \left(\frac{\int_{0}^{\infty} N_{Z} f\left(N_{Z}\right) d N_{Z}}{\int_{N_{\min }}^{\infty} N_{H I} f\left(N_{H I}\right) d N_{H I}}\right)-\log \left(N_{Z} / N_{H I}\right)_{\odot},
$$

where $N_{Z}$ and $N_{H I}$ are the column densities of metals and neutral hydrogen gas. Affected by the dust obscuration bias, nearly all the known DLAs lie below the threshold $\left(N_{Z n I I}=\right.$ $1.4 \times 10^{13} \mathrm{~cm}^{-2}$ ) introduced by Boissé et al. (1998). Therefore, most previous surveys (e.g. Kulkarni \& Fall 2002; Prochaska et al. 2003) suggest the mean metallicity measured by DLAs is $\langle Z\rangle \sim-1.5$ at a redshift $z \sim 2$, which is significantly lower than the metallicity $Z>-1.0$ measured in the Lyman break galaxies at the similar redshift (Giavalisco 2002; Fynbo et al. 2008) or other starlight emission-selected galaxies. This superficial "missing metals problem" might be solved by taking into account the significant population of dust obscured DLAs bearing bulks of heavy elements. Note that the metallicity of J1156+6155, $Z \sim-0.6$, is consistent with the measurement in emission-selected galaxies at $z \sim 2$. The dusty DLA population might be an important metal reservoir in the high redshift universe.

\footnotetext{
${ }^{9}$ It is beyond the scope of this paper to measure the number density.
} 
DLA J1157+6155 has a MW-like extinction curve with $2175 \AA$ dust extinction bump and MW-like dust depletion patterns and MW-like metallicity. The three features suggests that its counterpart galaxy may be an analog of the MW in the high redshift universe. Jiang et al. (2010b, 2011) detected three dozens of 2175- $\AA$ quasar absorbers with redshift of $z \sim 1.5$ in SDSS DR3. Most of these dusty quasar absorbers have strong metal absorption lines and thus may be metal-rich DLAs. In this paper, we have developed a more sensitive method to detected $2175 \AA$ bump on quasar spectra. We plan to apply the new method to the quasars in SDSS DR910 and expect to find more than 100 dusty absorbers with significant $2175 \AA$ extinction bump.

We would like to thank the anonymous referee for the enlightening suggestions that greatly improved the paper. We thank Yueheng Xu for helpful comments on the manuscripts. This work is supported by the Chinese NSF grant 10973012, 11033007 and 10973034, the SOC program CHINARE2012-02-03 and the National Basic Research Program of (973 Program) 2009CB824800. This work is also partially supported by NSF with grant NSF AST-0451407, AST-0451408 \& AST-0705139 and the University of Florida. This research has also been partially supported by the CAS/SAFEA International Partnership Program for Creative Research Teams. J. X. P. is supported by NSF grant (AST-0709235).

This work has made use of data obtained by the SDSS and MMT. The MMT telescope is operated by the MMT observatory, a joint venture of the smithsonian institution and the University of Arizona. Funding for the SDSS and SDSS-II has been provided by the Alfred P. Sloan Foundation, the Participating Institutions, the National Science Foundation, the US Department of Energy, the National Aeronautics and Space Administration, the Japanese Monbukagakusho, the Max Planck Society, and the Higher Education Funding Council for England. The SDSS Web Site is http://www.sdss.org//.

\section{REFERENCES}

Abazajian, K. N., et al. 2009, ApJS, 182, 543

Asplund, M., Grevesse, N., Sauval, A. J., \& Scott, P. 2009, ARA\&A, 47, 481

Bahcall, J. N., \& Peebles, P. J. E. 1969, ApJ, 156, L7

Boissé, P., Le Brun, V., Bergeron, J., \& Deharveng, J.-M. 1998, A\&A, 333, 841

\footnotetext{
${ }^{10}$ http://www.sdss3.org/dr9/
} 
Cleveland, W. S. 1979, J. Am. Stat. Assoc., 74, 829

Diplas, A., \& Savage, B. D. 1994, ApJ, 427, 274

Draine, B. T. 2003, ARA\&A, 41, 241

Elíasdóttir, Á., et al. 2009, ApJ, 697, 1725

Fall, S. M., \& Pei, Y. C. 1993, ApJ, 402, 479

Fall, S. M., \& Pei, Y. C. 1989, ApJ, 337, 7

Fall, S. M., Pei, Y. C., \& McMahon, R. G. 1989, ApJ, 341, L5

Fitzpatrick, E. L. 1999, PASP, 111, 63

Fitzpatrick, E. L., \& Massa, D. 1990, ApJS, 72, 163

Fitzpatrick, E. L., \& Massa, D. 2007, ApJ, 663, 320

Fynbo, J. P. U., Ledoux, C., Noterdaeme, P., et al. 2011, MNRAS, 413, 2481

Fynbo, J. P. U., Prochaska, J. X., Sommer-Larsen, J., Dessauges-Zavadsky, M., \& Møller, P. 2008, ApJ, 683, 321

Ge, J., \& Bechtold, J. 1997, ApJ, 477, L73

Ge, J., Bechtold, J., \& Kulkarni, V. P. 2001, ApJ, 547, L1

Giavalisco, M. 2002, ARA\&A, 40, 579

Gibson, R. R., et al. 2009, ApJ, 692, 758

Glikman, E., Helfand, D. J., \& White, R. L. 2006, ApJ, 640, 579

Gordon, K. D., Clayton, G. C., Misselt, K. A., Landolt, A. U., \& Wolff, M. J. 2003, ApJ, 594,279

Guimarães, R., Noterdaeme, P., Petitjean, P., Ledoux, C., Srianand, R., López, S. and Rahmani, H. 2012, AJ, 143, 147

Herbert-Fort, S., Prochaska, J. X., Dessauges-Zavadsky, M., Ellison, S. L., Howk, J. C., Wolfe, A. M., \& Prochter, G. E. 2006, PASP, 118, 1077

Hopkins, P. F., et al. 2004, AJ, 128, 1112 
Isobe, T., Feigelson, E. D., \& Nelson, P. I. 1986, ApJ, 306, 490

Jenkins, E. B. 1986, ApJ, 304, 739

Jiang, P., Ge, J., Zhou, H., Wang, J., \& Wang, T. 2011, ApJ, 732, 110

Jiang, P., Ge, J., Prochaska, J. X., Kulkarni, V. P., Lu, H. L., \& Zhou, H. Y. 2010a, ApJ, 720,328

Jiang, P., Ge, J., Prochaska, J. X., Wang, J., Zhou, H., \& Wang, T. 2010b, ApJ, 724, 1325

Junkkarinen, V. T., Cohen, R. D., Beaver, E. A., Burbidge, E. M., Lyons, R. W., \& Madejski, G. 2004, ApJ, 614, 658

Kaplan, K. F., Prochaska, J. X., Herbert-Fort, S., Ellison, S. L., \& Dessauges-Zavadsky, M. 2010, PASP, 122, 619

Kulkarni, V. P., Meiring, J., Som, D., Péroux, C., York, D. G., Khare, P., and Lauroesch, J. T. 2012, ApJ, 749, 176

Kulkarni, V. P., \& Fall, S. M. 2002, ApJ, 580, 732

Markwardt, C. B. 2009, Astronomical Society of the Pacific Conference Series, 411, 251

Meiring, J. D., et al. 2006, MNRAS, 370, 43

Nagamine, K., Springel, V., \& Hernquist, L. 2004, MNRAS, 348, 421

Noterdaeme, P., Petitjean, P., Ledoux, C., \& Srianand, R. 2009, A\&A, 505, 1087

Noterdaeme, P., Laursen, P., Petitjean, P., et al. 2012, A\&A, 540, A63

Noterdaeme, P., Ledoux, C., Petitjean, P., \& Srianand, R. 2008, A\&A, 481, 327

Pei, Y. C., Fall, S. M., \& Bechtold, J. 1991, ApJ, 378, 6

Pei, Y. C. 1992, ApJ, 395, 130

Péroux, C., McMahon, R. G., Storrie-Lombardi, L. J., \& Irwin, M. J. 2003, MNRAS, 346, 1103

Péroux, C., Dessauges-Zavadsky, M., D’Odorico, S., Sun Kim, T., \& McMahon, R. G. 2005, MNRAS, 363, 479

Pettini, M., Smith, L. J., Hunstead, R. W., \& King, D. L. 1994, ApJ, 426, 79 
Pettini, M., Smith, L. J., King, D. L., \& Hunstead, R. W. 1997, ApJ, 486, 665

Pitman, K. M., Clayton, G. C., \& Gordon, K. D. 2000, PASP, 112, 537

Prochaska, J. X., \& Wolfe, A. M. 1996, ApJ, 470, 403

Prochaska, J. X., Herbert-Fort, S., \& Wolfe, A. M. 2005, ApJ, 635, 123

Prochaska, J. X., \& Wolfe, A. M. 2009, ApJ, 696, 1543

Prochaska, J. X., Gawiser, E., Wolfe, A. M., Castro, S., \& Djorgovski, S. G. 2003, ApJ, 595, L9

Prochaska, J. X. 2006, ApJ, 650, 272

Prochaska, J. X., Wolfe, A. M., Howk, J. C., Gawiser, E., Burles, S. M. and Cooke, J. 2007, ApJS, 171, 29

Richards, G. T., et al. 2004, ApJS, 155, 257

Richards, G. T., Fan, X., Newberg, H. J., et al. 2002, AJ, 123, 2945

Roth, K. C., \& Blades, J. C. 1995, ApJ, 445, L95

Savage, B. D., \& Sembach, K. R. 1991, ApJ, 379, 245

Savage, B. D., \& Sembach, K. R. 1996, ARA\&A, 34, 279

Scaringi, S., Cottis, C. E., Knigge, C., \& Goad, M. R. 2009, MNRAS, 399, 2231

Schady, P., Savaglio, S., Krühler, T., Greiner, J., \& Rau, A. 2011, A\&A, 525, A113

Schlegel, D. J., Finkbeiner, D. P., \& Davis, M. 1998, ApJ, 500, 525

Schneider, D. P., et al. 2010, AJ, 139, 2360

Shen, Y., Richards, G. T., Strauss, M. A., et al. 2011, ApJS, 194, 45

Skrutskie, M. F., et al. 2006, AJ, 131, 1163

Tielens, A. G. G. M. 2005, The Physics and Chemistry of the Interstellar Medium, by A. G. G. M. Tielens, pp. . ISBN 0521826349. Cambridge, UK: Cambridge University Press, 2005

Tsuzuki, Y., Kawara, K., Yoshii, Y., Oyabu, S., Tanabé, T., \& Matsuoka, Y. 2006, ApJ, 650, 57 
Vanden Berk, D. E., et al. 2001, AJ, 122, 549

Vestergaard, M., \& Wilkes, B. J. 2001, ApJS, 134, 1

Vladilo, G. 2002, A\&A, 391, 407

Vladilo, G., Prochaska, J. X., \& Wolfe, A. M. 2008, A\&A, 478, 701

Wang, J., Hall, P. B., Ge, J., Li, A., \& Schneider, D. P. 2004, ApJ, 609, 589

Wang, J.-G., et al. 2009, ApJ, 707, 1334

Wolfe, A. M., Gawiser, E., \& Prochaska, J. X. 2005, ARA\&A, 43, 861

Xiao, T., Wang, T., Wang, H., Zhou, H., Lu, H. and Dong, X. 2012, MNRAS, 421, 486

Zhou, H., Ge, J., Lu, H., Wang, T., Yuan, W., Jiang, P., \& Shan, H. 2010, ApJ, 708, 742 


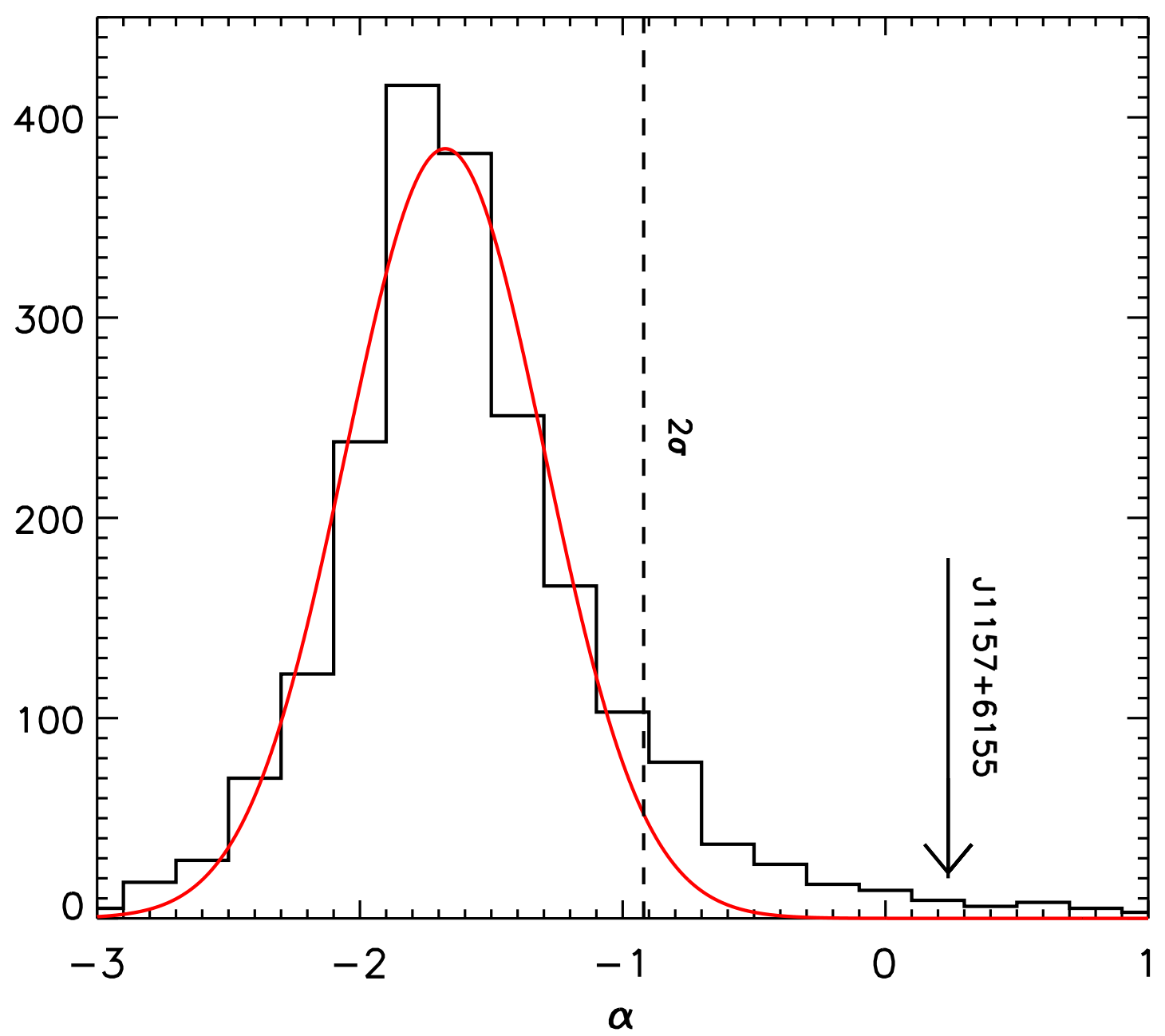

Fig. 1.- Distribution of the spectral indices $\alpha$ (defined as $S_{\lambda} \propto \lambda^{\alpha}$ ) of quasars from the SDSS DR7 in the redshift interval $2.4 \leq z \leq 2.6$. Overlayed is a Gaussian curve fitted to the data. The vertical dashed line indicates the $2 \sigma$ deviation from the center of the distribution. The value for $\mathrm{J} 1157+6155$ is indicated by the arrow. 

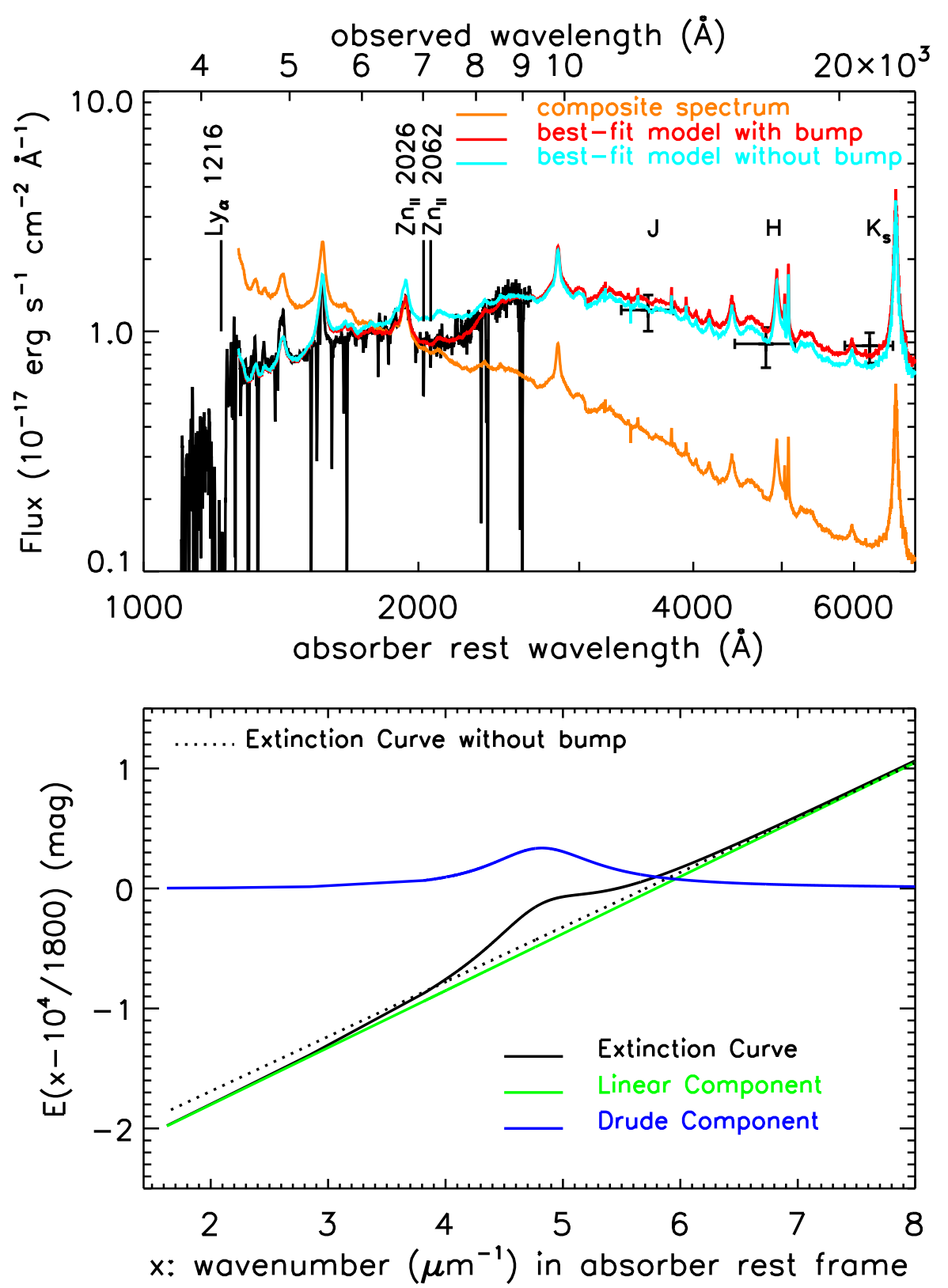

Fig. 2.- Upper: the observed data of J1157+6155. The observed spectrum is a combination of the MMT and SDSS spectra (black, smoothed by a 7-pixel boxcar). The composite quasar spectrum (orange) and the best-fit reddened model (red) are overplotted with the observed one. The 2MASS measurements in the $J, H$ and $K_{s}$ bands are consistent with the best-fit model. The cyan curve shows the model by reddening the composite quasar spectrum with a SMC-like extinction curve without a bump at around $2100 \AA$ in the DLA rest frame. Lower: The best-fit reddening curve (black solid) and its composites (green: a linear component; blue: a drude component). The fitted reddening curve without the bump was also plotted for comparison (dotted). 

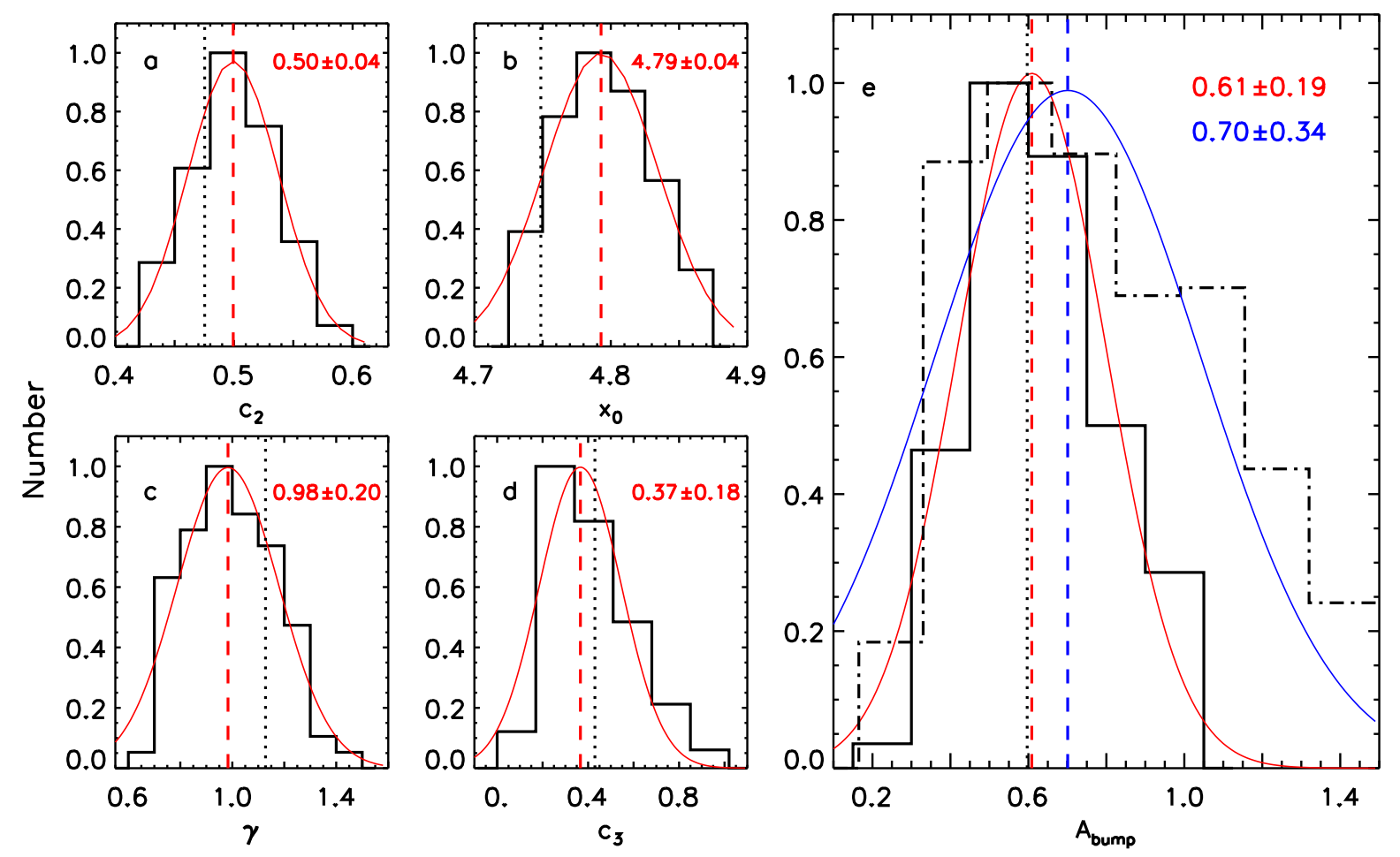

Fig. 3.- Panels $a, b, c, d$ : Distributions of the slope $c_{2}$, bump centroid $x_{0}$, bump width $\gamma$ and parameter $c_{3}$ of the extinction curve estimated from Fe II matched template spectra (solid histogram) and a Gaussian fit to them (red). The red dashed vertical lines show the best-fit results. The dotted vertical lines show the best-fit results of the composite quasar spectra method. Panel $e$ : Comparison of the bump strengths derived with the Fe II matched template spectra (in red) and with the full template library (in blue). The blue Gaussian is nearly twice broader than the red Gaussian, indicating that the refined modeling procedure is necessary for detecting relatively weak $2175 \AA$ absorption bumps on quasar spectra. See the text for details. 


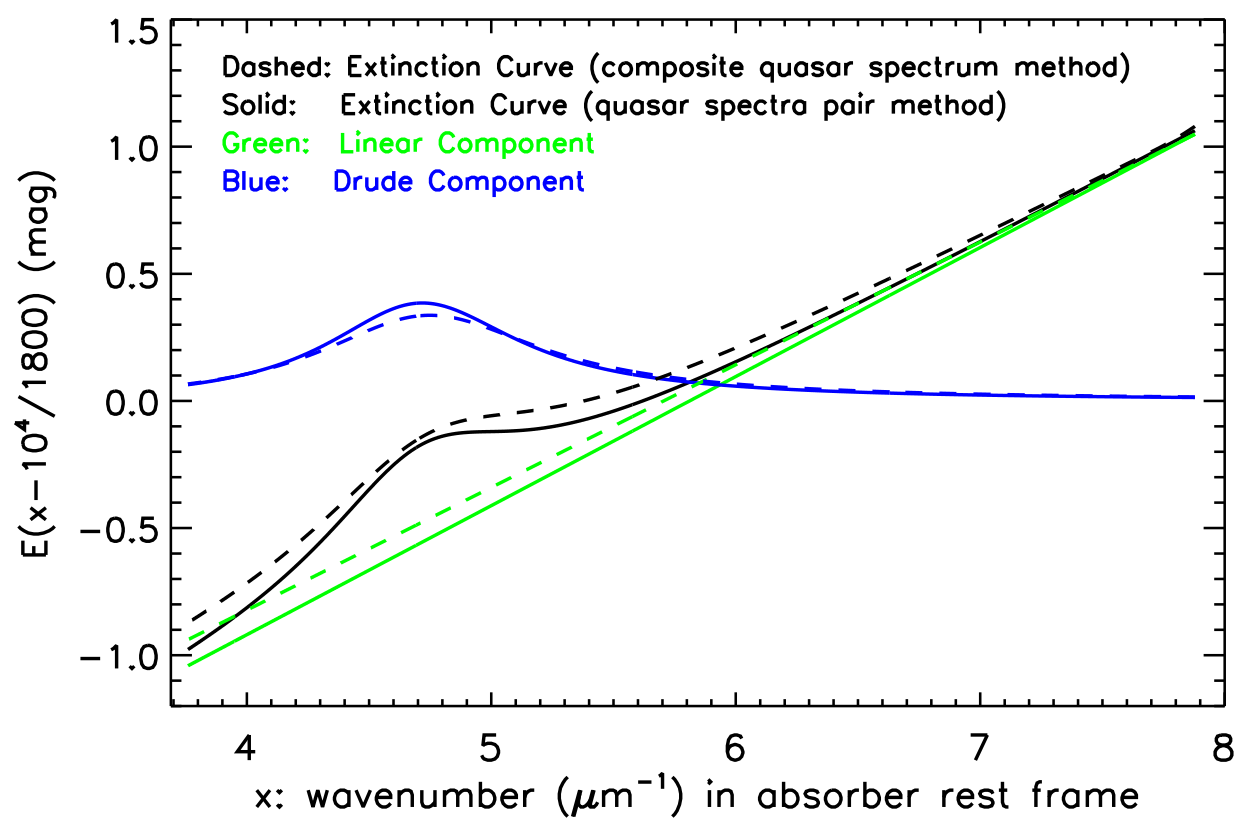

Fig. 4.- The comparison of extinction curve derived with quasar spectra pair method (solid) and that of the composite quasar spectrum method (dashed). 


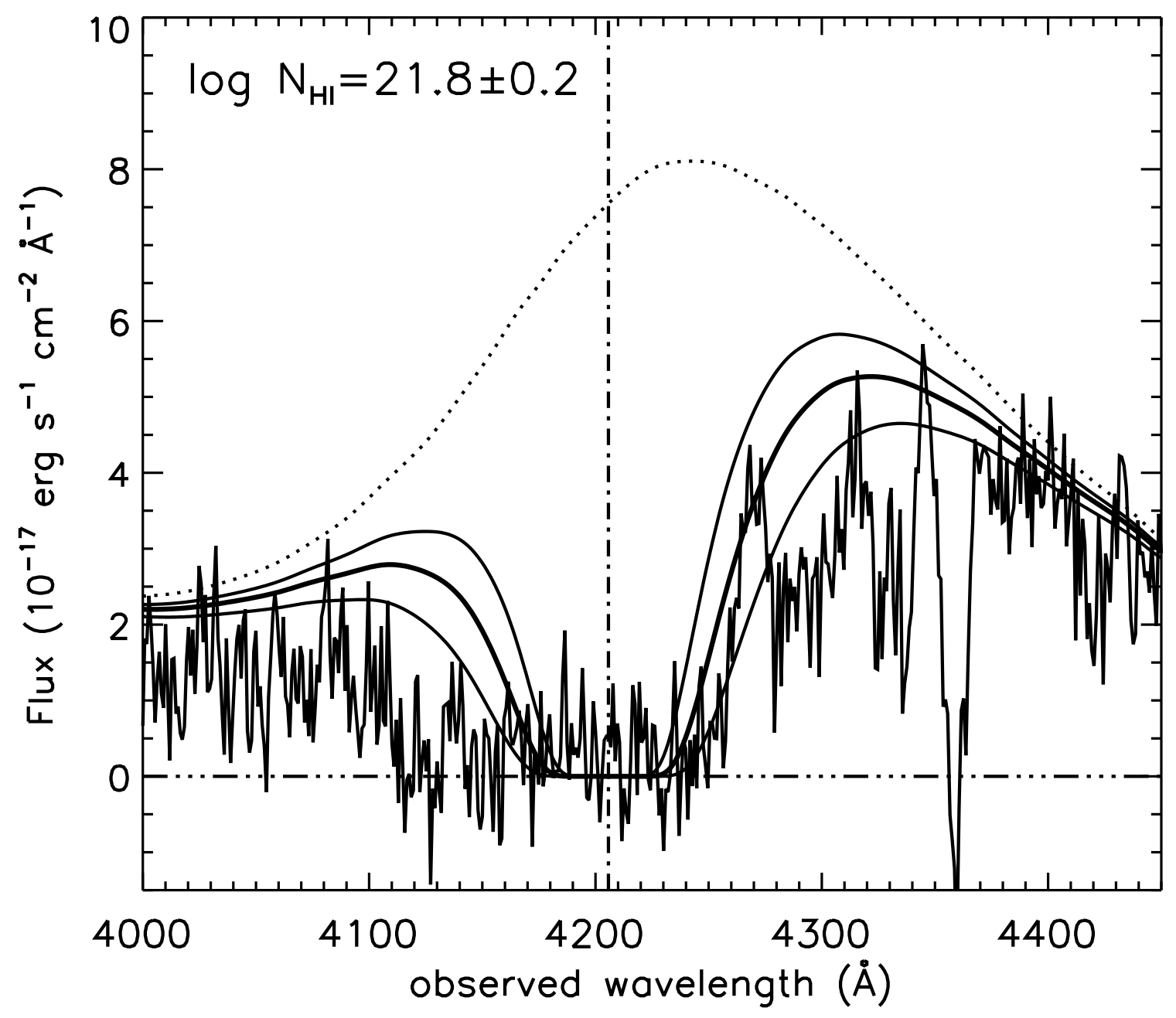

Fig. 5.- DLA line profile fitting on the MMT spectrum (black). The vertical dash-dotted line indicates the redshift estimated from narrow metal absorption lines. The dotted line is the predicted model of the quasar spectrum. The best-fit model is shown by the thick solid line, and the estimated $1 \sigma$ uncertainty is given by the thin solid lines. 


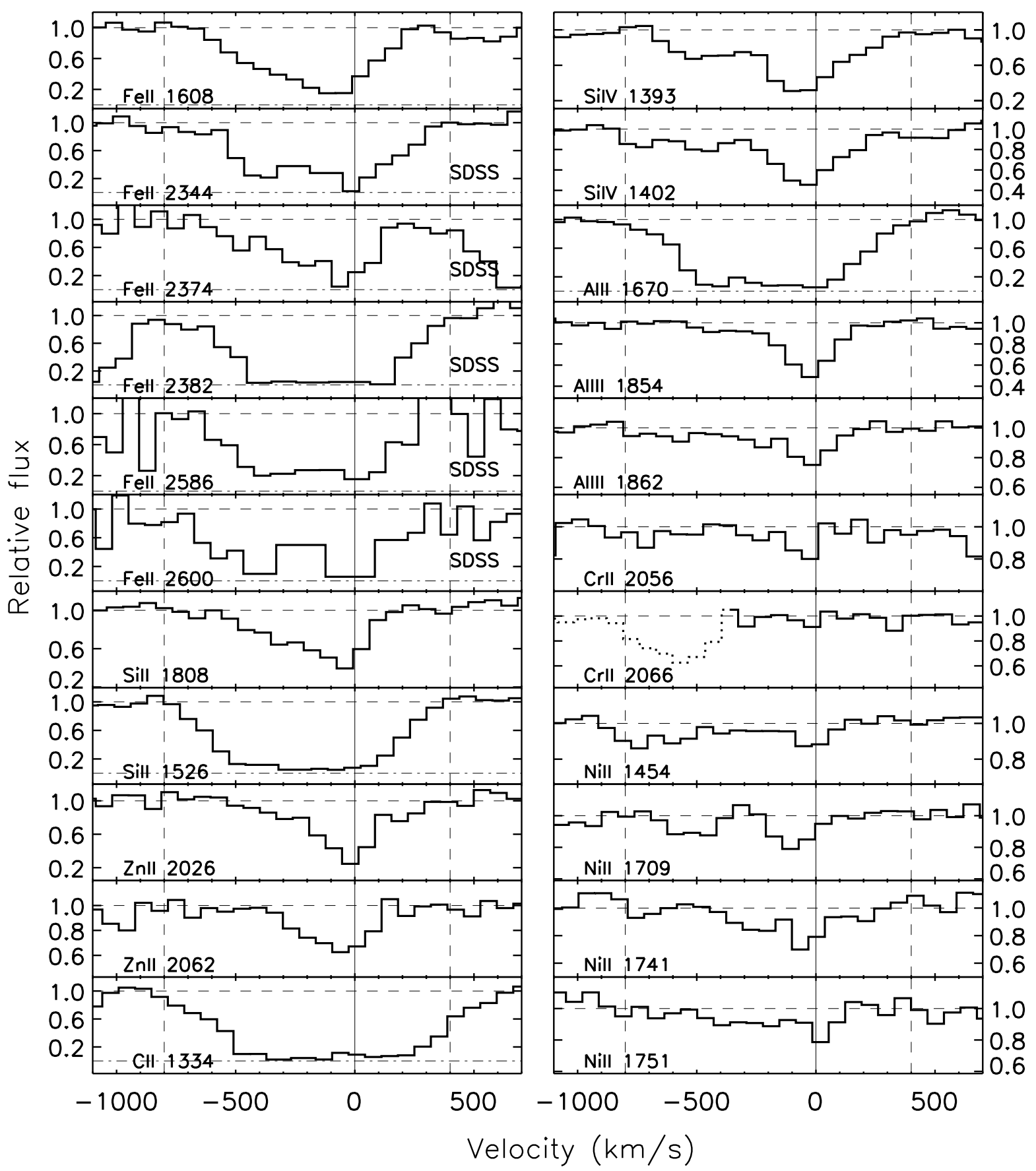

Fig. 6. - Normalized absorption spectrum of J1157+6155. The metal absorption lines are plotted in the velocity space. The zero velocity point (the solid line) is corresponding to $z_{a b s}=2.4596$. The two vertical dashed lines show the velocity interval used to measure the EW and $N_{A O D M}$. The Fe $\lambda \lambda 2344,2374,2382,2586,2600$ absorption lines are taken from SDSS spectrum because they are not covered by MMT spectrum, while the rest of the lines are measured from the MMT spectra. 

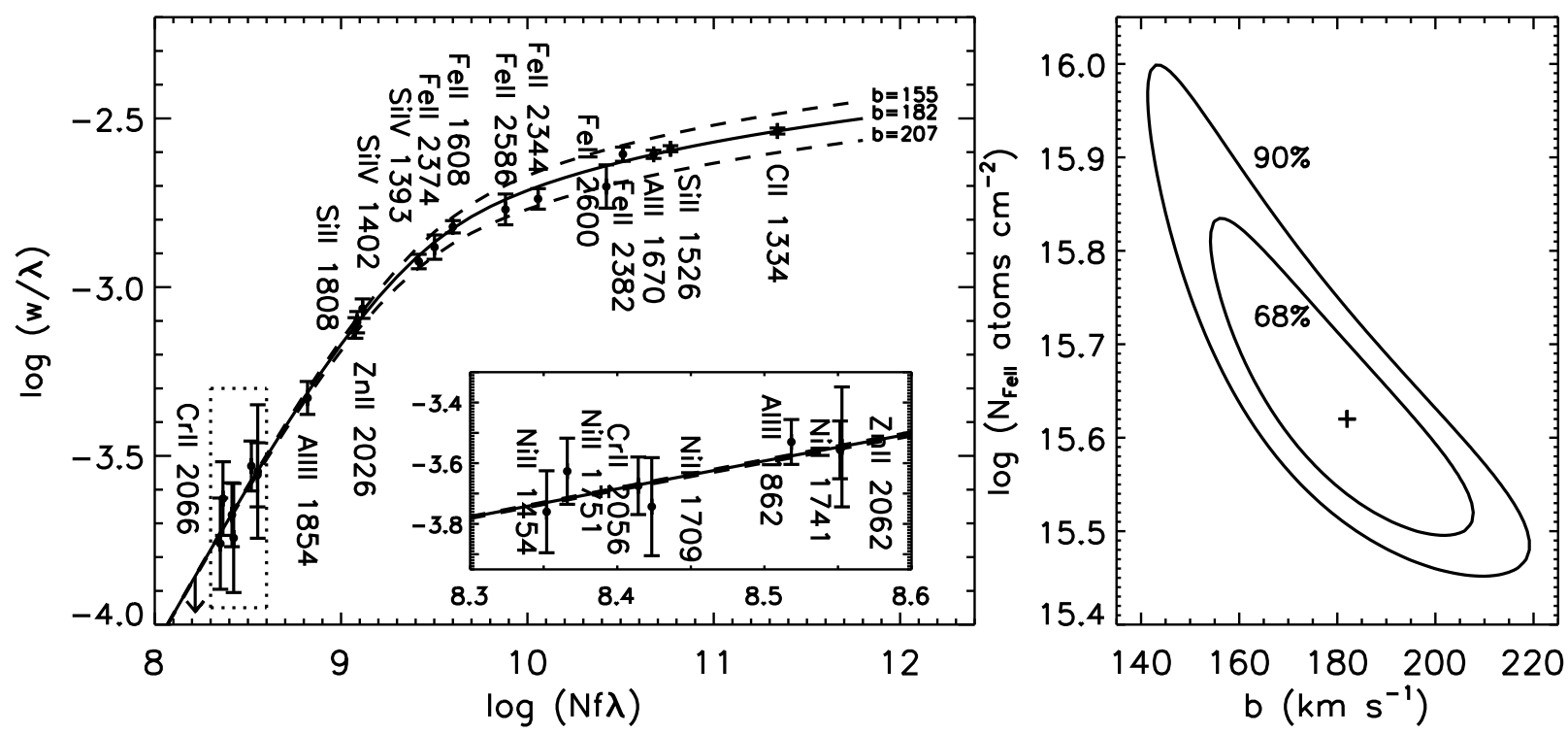

Fig. 7.- Left panel: Best-fit COG using six FeII absorption lines. The thick solid line represents the best-fit curve and the two dashed lines show the boundaries of the $68 \%$ confidence interval, as shown in the right panel. The dotted square is enlarged in the inserted. Right panel: $\chi^{2}$ contours in the $\log N_{F e I I}$-b plane. The minimum $\chi^{2}$ is marked as a cross. 


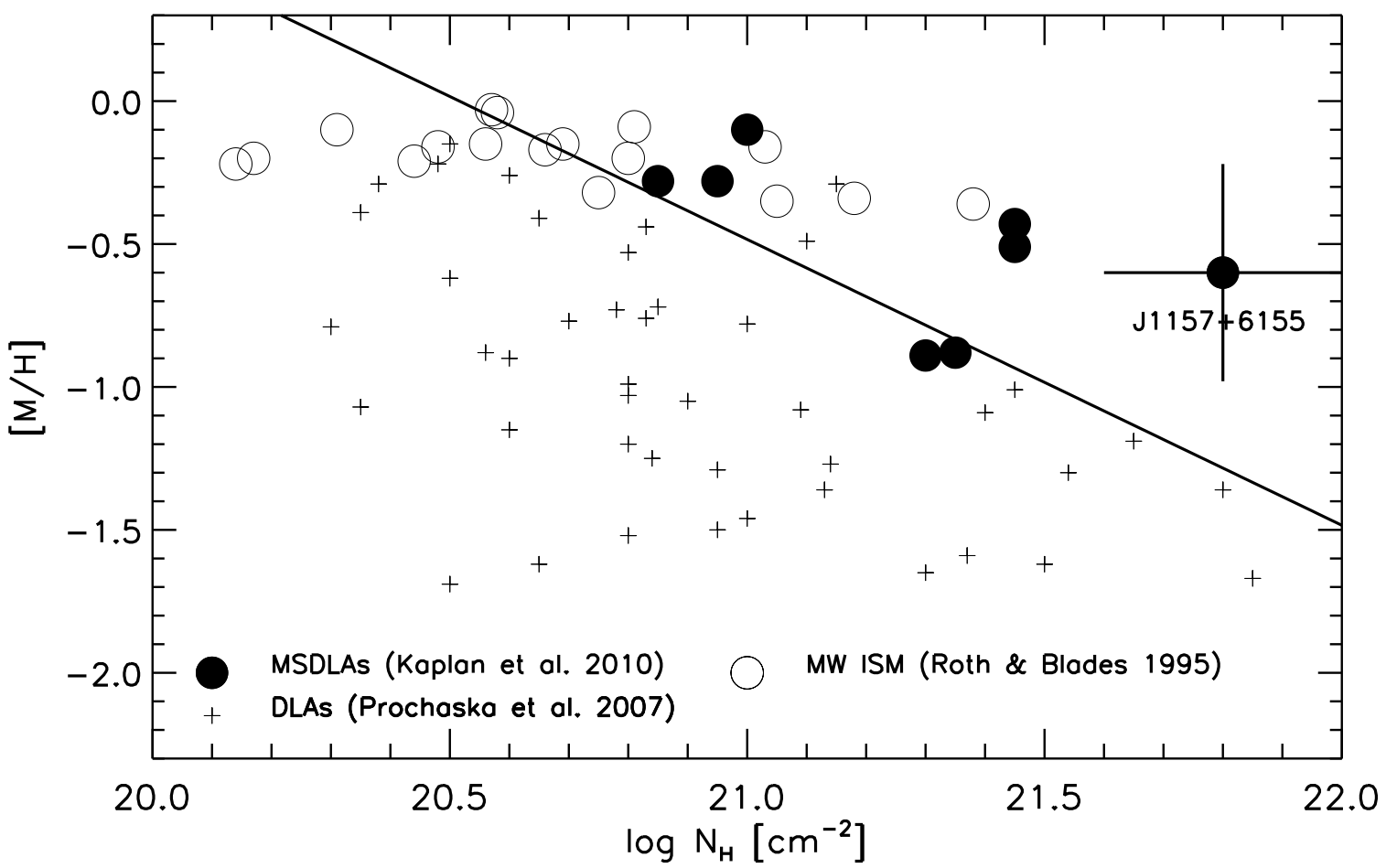

Fig. 8.- Metallicity in different absorbers. The tiny plus symbols are the normal DLAs in Prochaska et al. (2007). The $[\mathrm{M} / \mathrm{H}]$ of these objects are measured from $\mathrm{Zn}^{+}, \mathrm{Si}^{+}$or $S^{+}$. Nearly all of them are sitting under the dust obscuration threshold (the solid line) defined by Boissé et al. (1998). The open circles are the clouds in the MW, where the metallicity is measured by $[\mathrm{Zn} / \mathrm{H}]$ (Roth \& Blades 1995). The metal-strong DLAs (Kaplan et al. 2010), including J1157+6155 in this work, are labeled as filled circles. Most of them are located above the obscuration threshold and have similar metallicity $([\mathrm{Zn} / \mathrm{H}])$ with MW clouds. Especially, J1157+6155 have the highest column density of neutral hydrogen among the known metal-strong DLAs. 


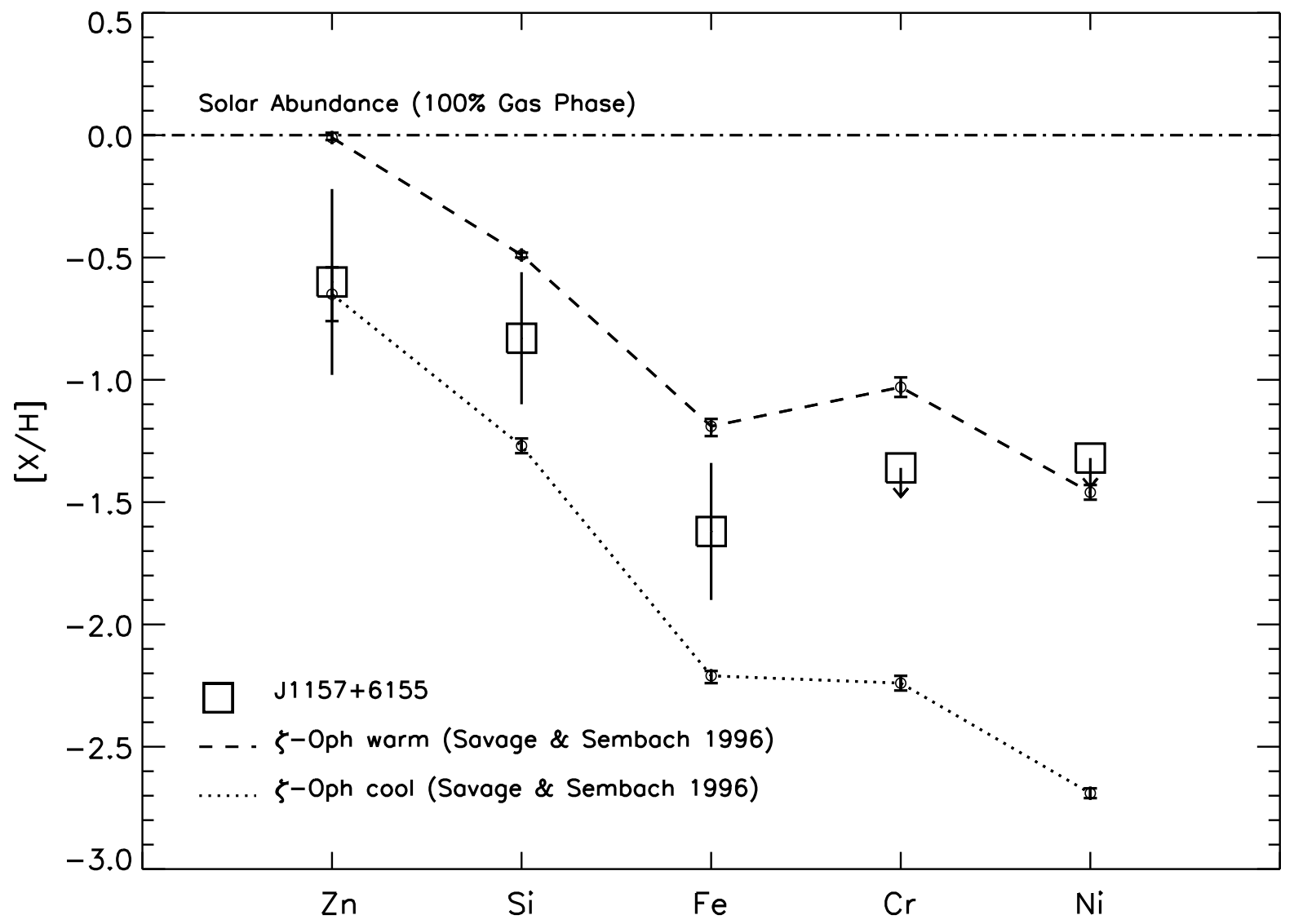

Fig. 9.- The dust depletion pattern of J1157+6155 is similar with the pattern measured in the MW, lying between that of the warm and cool clouds in the sight line toward $\zeta$ Oph. 


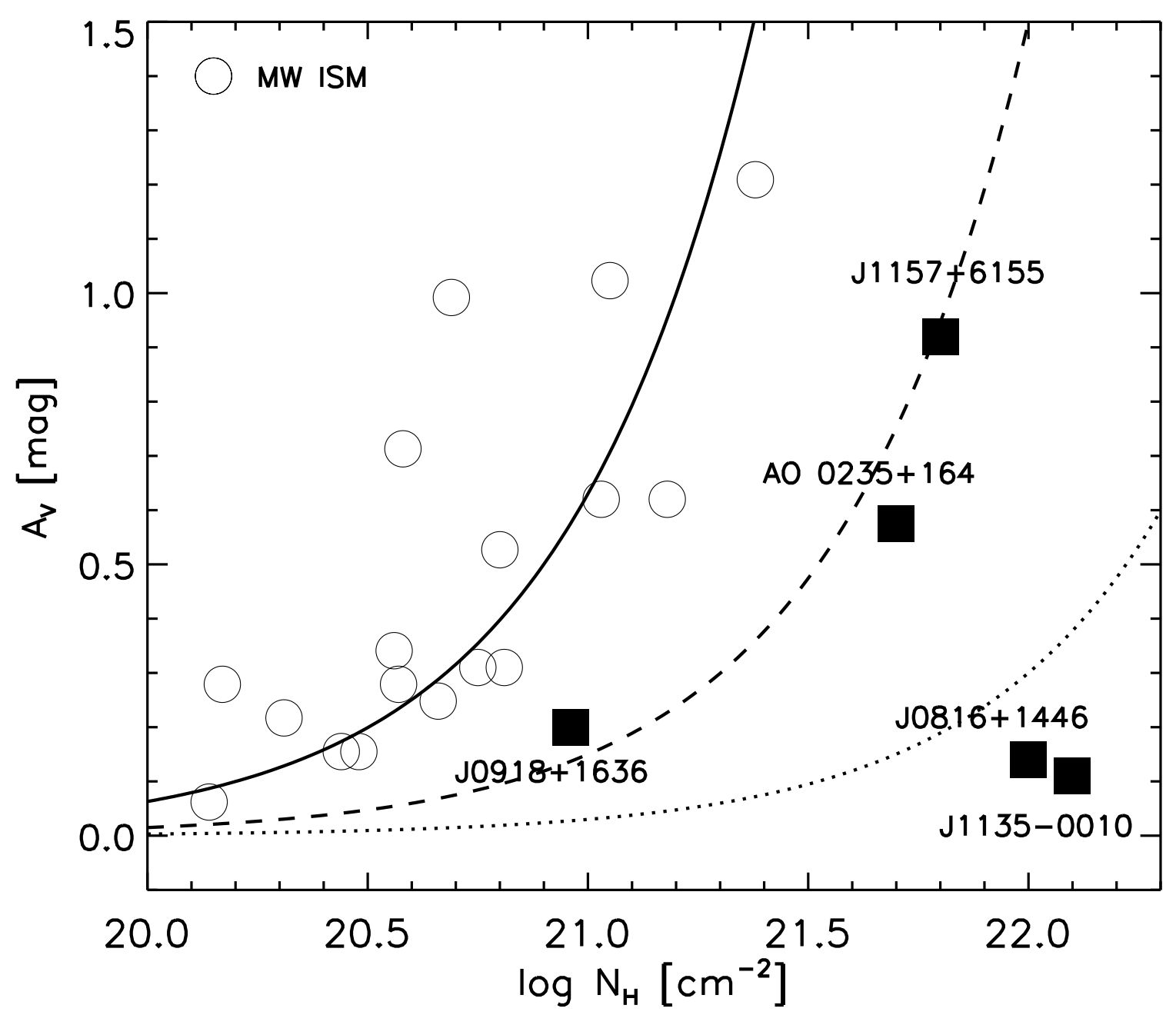

Fig. 10.- Diversity of the dust-to-gas ratio of DLAs. The dust-to-gas ratio of MW clouds are shown as open circles, the average value of which is $A_{V}=6.3 \times 10^{-22} N_{H}$ (the solid line; Diplas \& Savage 1994). The average dust-to-gas ratio of DLAs, $A_{V}=3 \times 10^{-23} N_{H}$ (the dotted line; Vladilo et al. 2008), is much smaller than that of MW clouds. Moreover, the dispersion of dust-to-gas ratios of DLAs is quite large. For example, the two super-DLAs (J0816+1446 and J1135-0010) have high column densities of gas but very little dust content. While the dust-to-gas ratio (the dashed line) of the three dust-rich DLAs (J0918+1636 and AO $0235+164$ as well as J1157+6155 in this work) is larger than the average ratio by a factor of 5 . The large extinction of J1157+6155, $A_{V} \sim 1.0$, has been seen in the most dusty MW clouds. 
Table 1. Ionic Column Densities for DLA J1157+6135

\begin{tabular}{cccccc}
\hline \hline Ion & $\begin{array}{c}\text { Transition } \\
(\AA)\end{array}$ & $\log f$ & $\begin{array}{c}\text { EW } \\
(\AA)\end{array}$ & $\begin{array}{c}\log N_{A O D M} \\
\left(\mathrm{~cm}^{-2}\right)\end{array}$ & $\begin{array}{c}\log N_{C O G} \\
\left(\mathrm{~cm}^{-2}\right)\end{array}$ \\
\hline \multirow{2}{*}{ Fe II } & 1608.4510 & -1.2366 & $2.43 \pm 0.14$ & $15.49 \pm 0.10$ & $15.63 \pm 0.19$ \\
& 2344.2141 & -0.9431 & $4.28 \pm 0.33$ & $15.16 \pm 0.11$ & $\ldots$ \\
& 2374.4612 & -1.5045 & $3.12 \pm 0.29$ & $15.55 \pm 0.12$ & $\ldots$ \\
& 2382.7649 & -0.4949 & $5.90 \pm 0.31$ & $14.93 \pm 0.13$ & $\ldots$ \\
& 2586.6499 & -1.1605 & $4.40 \pm 0.50$ & $15.27 \pm 0.13$ & $\ldots$ \\
Si II & 2600.1729 & -0.6216 & $5.17 \pm 0.83$ & $14.74 \pm 0.15$ & $\ldots$ \\
& 1808.0129 & -2.6603 & $1.37 \pm 0.14$ & $16.45 \pm 0.10$ & $16.48 \pm 0.18$ \\
Zn II & 1526.7065 & -0.8962 & $3.93 \pm 0.13$ & $15.58 \pm 0.11$ & $\ldots$ \\
& 2026.1360 & -0.3107 & $1.59 \pm 0.16^{a}$ & $14.10 \pm 0.11^{a}$ & $14.09 \pm 0.11$ \\
C II & 2062.6641 & -0.5918 & $0.91 \pm 0.15^{b}$ & $14.04 \pm 0.11^{b}$ & $13.83 \pm 0.33$ \\
Si IV & 1334.5323 & -0.8935 & $3.87 \pm 0.13$ & $15.69 \pm 0.11$ & $17.11 \pm 0.70$ \\
& 1393.7550 & -0.2774 & $1.66 \pm 0.13$ & $14.40 \pm 0.10$ & $14.55 \pm 0.12$ \\
Al II & 1402.7700 & -0.5817 & $1.21 \pm 0.13$ & $14.52 \pm 0.10$ & $\ldots$ \\
Al III & 1670.7874 & 0.2742 & $4.14 \pm 0.15$ & $14.32 \pm 0.11$ & $15.18 \pm 0.49$ \\
& 1854.7164 & -0.2684 & $0.87 \pm 0.14$ & $13.81 \pm 0.11$ & $13.82 \pm 0.12$ \\
Cr II & 1862.7896 & -0.5719 & $0.55 \pm 0.14$ & $13.86 \pm 0.12$ & $\ldots$ \\
& 2056.2539 & -0.9788 & $0.44 \pm 0.15$ & $14.07 \pm 0.15$ & $<14.08^{c}$ \\
Ni II & 2066.1609 & -1.2882 & $0.16 \pm 0.14$ & $13.82 \pm 0.35$ & $<14.19^{c}$ \\
& 1454.8420 & -1.4908 & $0.25 \pm 0.14$ & $14.70 \pm 0.16$ & $<14.68^{c}$ \\
& 1709.6042 & -1.4895 & $0.31 \pm 0.17$ & $14.61 \pm 0.20$ & $\ldots$ \\
& 1741.5531 & -1.3696 & $0.48 \pm 0.16$ & $14.70 \pm 0.13$ & $\ldots$ \\
\hline & 1751.9156 & -1.5575 & $0.41 \pm 0.15$ & $14.73 \pm 0.16$ & $\ldots$ \\
\hline
\end{tabular}

Note. - Equivalent width is measured in the absorber's rest frame at $z=2.4596$. The vacuum wavelengths and oscillator strength $f$ are adopted from the Atomic Data compiled by J. X. Prochaska (http://kingpin.ucsd.edu/ hiresdla/atomic.dat). All statistical uncertainties represent the $68 \%$ confidence interval. The errors of $N_{A O D M}$ include a systematic error of 0.10 dex due to the uncertainty of normalization. $N_{C O G}$ is derived by using the best-fit COG and assuming the same column densities of different transition of same ions; the errors include the errors caused by the uncertainties in EWs and Doppler parameter b, as well as a systematic error of 0.10 dex due to the uncertainty of normalization.

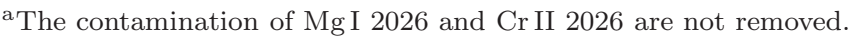

${ }^{\mathrm{b}}$ The contamination of CrII 2026 is not removed from EW and $N_{A O D M}$. When calculating $N_{C O G}$, the contamination of Cr II 2062 is removed by scaling the EW of Cr II 2056 according to their oscillator strengths.

${ }^{\mathrm{c}}$ Derived from the $3 \sigma$ upper limit of the EW. 